\title{
Estrategias competitivas utilizadas por las MIPYMES del sector servicio de comida buffet de la ciudad de Estelí en relación al marketing de servicio en el primer semestre 2016, Nicaragua
}

\author{
Arlen Meryfel Picado Juárez ${ }^{1}$ \\ Yasmina Ramírez Sobalvarro ${ }^{2}$ \\ Natalia Golovina ${ }^{3}$
}

\section{RESUMEN}

Este artículo es resultado de una investigación realizada en el primer semestre del año 2016, sobre seis empresas que participaron en el estudio, dedicadas a ofrecer el servicio de comida buffet en la ciudad de Estelí. El objetivo de investigación fue analizar las estrategias competitivas utilizadas por las MIPYMES del sector servicio de comida buffet de la ciudad de Estelí en relación al marketing de servicio en el primer semestre del año 2016. Se identificaron las estrategias y elementos del marketing de servicios utilizados por los propietarios del sector, se describió su implementación, se realizó el análisis de estrategias por medio de la técnica del diamante de Porter, en base a los resultados se propusieron estrategias competitivas. Esta investigación es con enfoque cualitativo con ciertos elementos cuantitativos, de tipo aplicada, paradigma interpretativo, de corte transaccional. Se utilizaron como estrategias de recolección de datos: entrevistas a los propietarios, encuestas a los clientes y guía de observación directa en los locales.

Palabras claves: Estrategias, competitividad, marketing de servicio, empresas.

Recibido: 27 de julio de 2016

Aceptado: 17 de octubre de 2016

1 Egresada de la maestría en Gestión Sostenibilidad y Calidad de las MIPYMES, docente en UNAN-Managua/ FAREM-Estelí. Correo electrónico: arlenmery@yahoo.com

2 Tutora de investigación, Máster en Dirección Estratégica de Marketing, docente en UNAN-Managua/FAREMEstelí. Correo electrónico: jazminaramirez@yahoo.com

3 Asesora metodológica. Doctora en Ciencias Sociales de la Universidad del Zulia. Docente en UNAN-Managua/ FAREM-Matagalpa. Correo electrónico: natygolovina@gmail.com 


\title{
Competitive strategies implemented by the SME's of the buffet sector in Estelí, in relation to the service market, during the first semester of 2016, Nicaragua
}

\begin{abstract}
This article derives from a research work conducted during the first semester of 2016, about six companies that participated in this study. The companies are dedicated to offering offer buffet food in Estelí. The main research objective was to analyze the competitive strategies implemented by the buffet sector in Estelí, during the first semester of 2016. The strategies and marketing elements used by the sector owners were identified. Moreover, their implementation was described. In addition, the strategies were analyzed by using the Porter-diamond technique, which served as a basis for proposing competitive strategies. This research followed a qualitative approach, and featured some quantitative elements. The research is applied and belongs to the interpretative paradigm. As data collection methods, interview to owners, surveys to customers and direct observation to the companies were used.
\end{abstract}

Key words: Strategies, competitiveness, service market, companies. 


\section{INTRODUCCIÓN}

El sector servicios de comida buffet forman parte de las micro, pequeñas y medianas empresas de nuestro país. En Nicaragua las MIPYMES generan casi 300 mil empleos, en más de 150 mil establecimientos, de los cuales $55 \%$ es administrado por mujeres, según datos del 2009 del Ministerio de Fomento, Industria y Comercio. En las zonas urbanas estas unidades económicas generan el 58\% del empleo, según datos del Directorio Económico Urbano del Banco Central de Nicaragua. Asociando a la flexibilidad de sus procesos productivos, convierte a estas empresas en un potencial de crecimiento y desarrollo económico (Díaz \& Sánchez, 2011)

En el departamento de Estelí han incrementado el número de empresas dedicadas a ofrecer el servicio de comida buffet, el rubro alimentos es uno de los más difíciles, debido a que se tiene que cuidar hasta el último detalle. Existen enormes riesgos de que la comida salga mal, que se afecte la salud del consumidor, que el servicio no sea el esperado o simplemente que se prepare inadecuadamente. La comida es un negocio vivo, y como tal, es impredecible y riesgoso.

En la actualidad, los cambios en los hábitos y las formas alimenticias son parte de una tendencia global de mayor integración. Las cocinas son mucho más exactas en cuanto a su origen étnico y regional, cocinas eclécticas que ofrecen nuevas perspectivas en cuanto a estilo y sabor, variaciones audaces sobre las costumbres del comer. Cada día, los consumidores se abren a nuevas posibilidades de alimentación y aceptan infinidad de propuestas.

El vice presidente de la Cámara de Industrias de Nicaragua, Mario Amador, declaró que las microempresas requieren mejorar su competitividad. "Para esto se necesita potenciar las capacidades gerenciales, que estos sectores puedan conocer sus oportunidades y puedan contar con todas las herramientas de los conocimientos técnicos para la administración de los negocios" (2013).

El estudio de la competitividad de las MIPYMES nicaragüenses es de suma importancia, estas empresas se instalan en el mercado nacional, crecen según sus posibilidades pero en ese desarrollo llegan a presentar diversas problemáticas, es por ello, que en esta investigación se plantea la pregunta problema ¿Cuáles son las estrategias competitivas que utilizan las MIPYMES del sector servicio de comida buffet de la ciudad de Estelí en relación al marketing de servicio en el primer semestre del año 2016?, la que permitirá concretar la existencia de problemas y necesidades reales de estas empresas.

\section{MATERIALES Y MÉTODOS}

De acuerdo al problema, propósito y objetivos de investigación, es un estudio cualitativo con ciertos elementos cuantitativos. El paradigma de investigación según el propósito es interpretativo, busca comprender la realidad sobre la aplicación de estrategias competitivas y el marketing de servicio en las MIPYMES del sector servicio de comida buffet.

Para obtener los resultados de la investigación se hizo uso de preguntas de contenido y por lo que además se empleará enfoque cuantitativo que permitirá identificar los beneficios que han obtenido las MIPYMES del sector servicio de comida buffet con la utilización de estrategias competitivas y los elementos del marketing de servicio, las problemáticas, la competencia en el sector.

La investigación cualitativa se enfoca a comprender y profundizar los fenómenos, explorándolos desde la perspectiva de los participantes en un ambiente natural y en relación con el contexto. El enfoque cualitativo se selecciona cuando se busca comprender la perspectiva de los participantes (individuos o grupos pequeños de personas a los que se investigará) acerca 
de los fenómenos que los rodean, profundizar en sus experiencias, perspectivas, opiniones y significados, es decir, la forma en que los participantes perciben subjetivamente su realidad. (2010, pág. 364)

Según el propósito del estudio es de tipo descriptivo y explicativo, ya que se determinará si las MIPYMES del sector servicio de comida buffet de la ciudad de Estelí implementan estrategias competitivas y elementos del marketing de servicio y la competitividad de las empresas en estudio.

De acuerdo al nivel de investigación es aplicada; al analizar el problema se darán soluciones, mediante aportes concretos, tales como la propuesta de estrategias que contribuyan a la competitividad de las MIPYMES en estudio.

Según el alcance el estudio es de corte transaccional en marcada en un periodo de tiempo determinado, donde se determinara la utilización de estrategias competitivas con respecto al marketing de servicio en el primer semestre de 2016.

La población está conformada por 10 establecimientos de cuales 6 aceptaron participar en el estudio, estos con categoría de un tenedor a cinco tenedores (lista de establecimientos proporcionados por el INTUR, Estelí). También se incluye en la población a los clientes que concurren a dichos establecimientos a continuación tabla:

Tabla $N^{0}$ 1: Lista de buffet en la ciudad de Estelí

\begin{tabular}{llll}
\hline $\mathbf{N}^{\circ}$ & $\begin{array}{c}\text { Nombre } \\
\text { establecimiento }\end{array}$ & Actividad & Categoría 2015 \\
\hline 1 & Buffet Estelí & Restaurante & Un tenedor \\
2 & Don Juan & Restaurante & Un tenedor \\
3 & Chaman & Restaurante & Un tenedor \\
4 & Sazón Norteño & Restaurante & Dos tenedores \\
5 & Cándida & Restaurante & Dos tenedores \\
6 & Castillo's Buffet & Restaurante & Tres tenedores \\
\hline
\end{tabular}

Fuente: Lista de MIPYMES de servicios Buffet en la ciudad de Estelí, proporcionada por Lic. Marden Benavidez, responsable de inspección y registro de INTUR, Estelí
La muestra se determinó en base al muestreo no probabilístico utilizado en las investigaciones cualitativas.

El muestreo a utilizar es por conveniencia; ya que se trabajó con las MIPYMES del sector servicio de buffet que estuvieron dispuestas a participar en el proceso de investigación.

El muestreo a utilizar en la población de clientes está determinado por la cantidad de consumidores que concurren a estos establecimientos, diariamente asisten 795, en los seis locales, los datos en la siguiente tabla:

Tabla $\mathbf{N}^{\circ}$ 2: Cantidad de clientes por establecimiento

Nombre del
establecimiento Cometarios según entrevista

\begin{tabular}{|c|c|}
\hline Buffet Estelí & $\begin{array}{l}\text { "Aproximadamente se atienden } 250 \\
\text { clientes diario". (Alvares, 2016) }\end{array}$ \\
\hline Don Juan & $\begin{array}{l}\text { "Atendemos aproximadamente } 200 \\
\text { clientes al día". (Sevilla, 2016) }\end{array}$ \\
\hline Chaman & $\begin{array}{l}\text { "Se atienden } 100 \text { clientes diariamente". } \\
\text { (Reyes, 2016) }\end{array}$ \\
\hline Sazón Norteño & $\begin{array}{l}\text { "Se atienden } 100 \text { clientes diario". } \\
\text { (Gutiérrez, 2016) }\end{array}$ \\
\hline Cándida & $\begin{array}{l}\text { "Llegan diariamente } 70 \text { clientes". } \\
\text { (Orozco, 2016) }\end{array}$ \\
\hline Castillo's Buffet & $\begin{array}{l}\text { "Vienen } 75 \text { clientes cada día". (Ruiz, } \\
\text { 2016) }\end{array}$ \\
\hline
\end{tabular}

El cálculo se realizó tomando en cuenta la disposición de cada MIPYME que se dispuso a participar en el proceso de investigación, para ello se consultó al propietario la cantidad aproximada de clientes que consumen diariamente. Este cálculo se realizó para aplicar las encuestas dirigidas a los clientes.

La recolección de información permitirá dar cumplimiento de los objetivos planteados; están: entrevistas dirigidas a los propietarios, encuestas a los clientes y guía de observación.

Para obtener la información de los propietarios y consumidores de las MIPYMES del sector de comida 
buffet se aplicarán: las entrevistas, encuestas, y guía observación. Es importante destacar que se trabajó con las MIPYMES que se dispusieron a participar de forma voluntaria en el proceso de investigación. Posterior al trabajo de campo se realizó el análisis de la información, este análisis reflejó los resultados dando respuesta a los objetivos propuestos.

La entrevista se analizó por objetivos de investigación y las encuestas se procesaran utilizando el software estadístico SPSS versión 20, realizando un análisis descriptivo-explicativo, se realizará cruce de algunas variables, los cuales se reflejarán en tablas y gráficos.

La observación complementó el análisis de investigación, ya que, comprobó las condiciones de los locales, vestimenta del personal, los productos ofrecidos, la cantidad de consumidores que concurren a los locales, entre otros elementos de análisis planteados en la matriz de objetivos. Este análisis se reflejó en los resultados por objetivos, con este instrumento se realizó análisis de correspondencia entre variables dependientes.

El análisis de correspondencias es una técnica estadística para analizar la relación entre categorías de variables cualitativas. Es una técnica de "reducción de dimensiones en el contexto de tablas de contingencia. El propósito es representar gráficamente la estructura de relaciones de dos o más variables cualitativas mediante mapas de posicionamiento". El análisis de correspondencias múltiples, a diferencia del análisis de correspondencias simple, trabaja con más de 2 variables, lo que permite incluir variables objetos, que son aquellas donde cada categoría tiene frecuencia igual a 1 (como países o comunas).

Al igual que en el análisis de correspondencias simples, las relaciones entre las categorías de las variables, se analizan a través de los mapas perceptuales, donde cada categoría es ubicada en un punto. La distancia entre las distintas categorías nos permitirá analizar la relación entre ellas. (Díaz \& Garrido, 2015)

\section{RESULTADOS Y DISCUSIÓN}

Se detallan los principales resultados obtenidos de la aplicación de técnicas de recolección de datos (entrevista estructurada a propietarios, encuesta dirigida a los clientes y observación en los establecimientos), así como la triangulación de estos datos.

A continuación se realiza una breve caracterización del sector en estudio.

Las MIPYMES del sector servicio de comida buffet en la ciudad de Estelí está conformada por 10 establecimientos, de las cuales 7 cuentan con categoría de uno a cinco tenedores (registro del INTUR, ver anexo $\mathrm{N}^{\mathrm{o}} 05$ ) de las cuales 6 de aceptaron participar en el estudio.

De estas seis empresas, cuatro son dirigidas por mujeres y dos por varones, propietarios y propietarias de las edades comprendidas entre 35 a 49 años, con formación universitaria. Los años de funcionamiento son variados, van desde 1 año del establecimiento más reciente a 17 años del establecimiento más viejo. La cantidad de trabajadores está comprendida de 5 a 15 trabajadores.

A continuación se muestran los resultados por objetivo de seis empresas del sector en estudio:

Objetivo número 1: Determinar las estrategias competitivas utilizadas por las MIPYMES del sector servicio de comida buffet de la ciudad de Estelí.

En las encuestas, los clientes declararon que los propietarios de los seis establecimientos utilizan estrategias competitivas 
Gráfico 1: El propietario utiliza estrategia para atraer al cliente

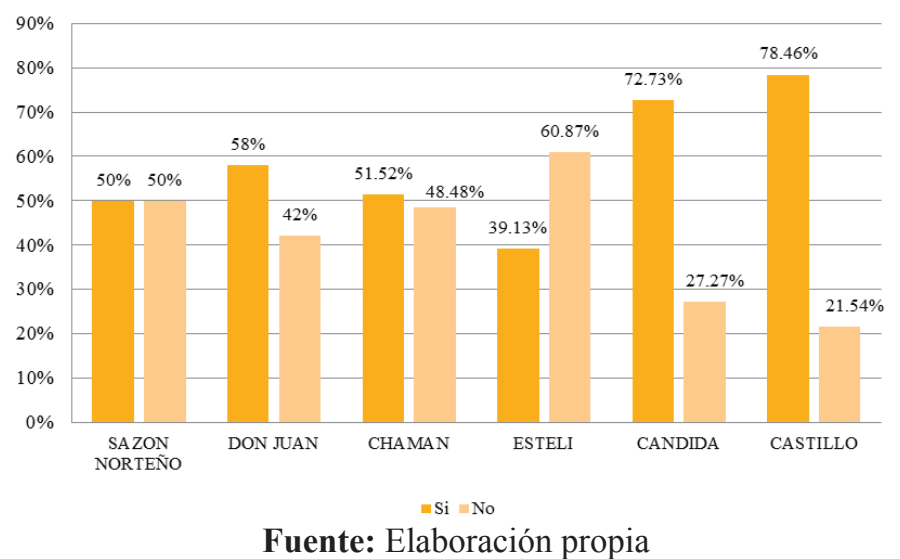

En el gráfico número 1, se visualiza que los clientes identifican que los propietarios utilizan estrategias para atraerlos al negocio. En el buffet Castillo con un porcentaje de $78.46 \%$ afirmaron la aplicación de estrategias, en tanto en el comedor Cándida el porcentaje es de $72.73 \%$ afirman que aplican estrategias, en el buffet Don Juan el porcentaje es de 58\% afirman la aplicación de estrategias, en el menor de los casos están: el buffet Chaman con un porcentaje de $52.51 \%$ afirman la aplicación de estrategias, en el Sazón norteño con un porcentaje de $50 \%$ afirman que si aplican estrategias y el buffet Estelí con un porcentaje de 39.13\% afirman la aplicación de estrategias. El cliente esta consiente que los propietarios están realizando esfuerzos para atraer clientela, los cuales son importantes para el desarrollo del negocio.

Se consultó a los propietarios si utilizan estrategias competitivas en sus negocios, los comentarios descritos a continuación:

Tabla $N^{\circ}$ 3: Implementación de estrategias competitivas

\begin{tabular}{cl}
\hline $\begin{array}{c}\text { Nombre del } \\
\text { establecimiento }\end{array}$ & \multicolumn{1}{c}{ Cometarios según entrevista } \\
\hline "Si, le brindamos un café de cortesía \\
Buffet Don Juan & $\begin{array}{l}\text { o a veces se trata de adecuar el plato } \\
\text { a la economía del cliente". (Alvares, } \\
2016)\end{array}$
\end{tabular}

Comedor

Cándida

Buffet Chaman

Buffet Estelí

Buffet Sazón norteño
Castillo's buffet

"Si, la calidad de nuestros productos y el precio, son nuestras dos mejores estrategias. La atención al cliente es un agregado ya que para nosotros es normal atenderlos bien". (Sevilla, 2016)

"Si, variamos el menú, mantenemos los precios, sacrificamos las utilidades un poco para mantener los precios y la remodelación del local". (Reyes, 2016)

"Si, adaptamos los platos al bolsillo de los clientes, cuando vienen grupos grandes les ofrecemos un plato de cortesía, ejemplo vienen siete personas pagan seis y uno le sale gratis". (Gutiérrez, 2016)

"Buena atención, un chocolate en un día especial". (Orozco, 2016)

"Ofreciendo buena calidad y un buen precio. Ponemos televisión y música instrumental". (Ruiz, 2016)

Fuente: Elaboración propia

En las entrevistas realizadas a los seis propietarios de los establecimientos de comida buffet, todos afirmaron utilizar estrategias competitivas en sus negocios, las que van desde ofrecer café de cortesía, adecuación del precio a las necesidades del cliente, productos de calidad, el precio de los productos, variedad en el menú, remodelación del local, platos de cortesía cuando llegan grupos grandes, buena atención hasta los dulces de cortesía en días especiales

Otro tipo de estrategias utilizadas por los propietarios para atraer a los clientes, es a través de las condiciones de los locales, a continuación los resultados según las encuestas: 
Gráfico 2: Condiciones del buffet

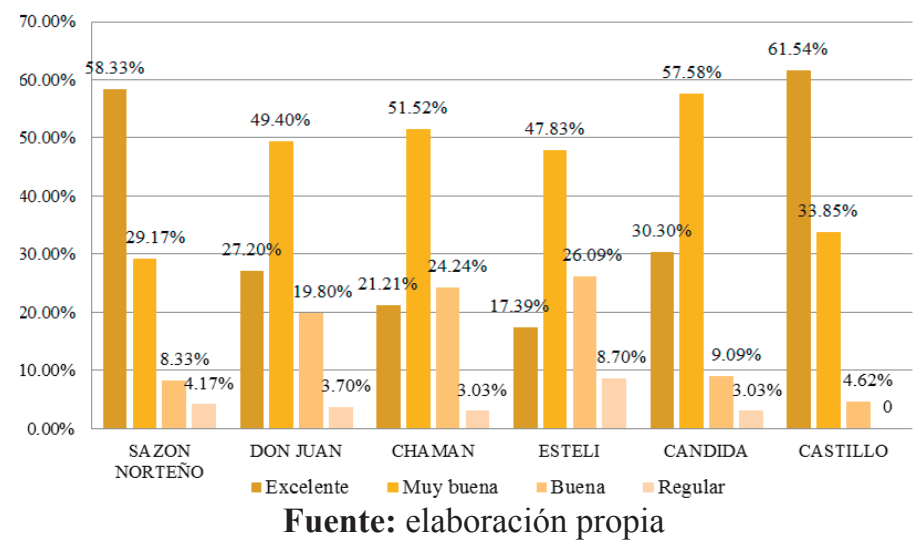

En el gráfico número 2, se observar que en cada establecimiento esta diferenciado en porcentajes, los clientes afirmaron que cinco locales tienen condiciones de comodidad, tales como: el buffet Sazón norteño con un porcentaje de $58.33 \%$ de excelente , en el buffet don Juan con un porcentaje de $49.40 \%$ de muy buena, el buffet Chaman con un porcentaje de $51.52 \%$ de excelente, el buffet Estelí con un porcentaje de $47.83 \%$ de muy buena y el comedor Cándida con un porcentaje $57.58 \%$ de muy buena, mientas que en el Castillo's buffet, los clientes afirman que las condiciones son excelentes con un porcentaje del $61.54 \%$.

Para confirmar este hecho, se consultó al cliente cuales son las condiciones de comodidad tienen los locales, a continuación los datos según encuesta:

Tabla $N^{\circ}$ 4: Condiciones que tienen los negocios

\begin{tabular}{|c|c|c|c|c|c|c|}
\hline \multirow{2}{*}{ Condiciones del negocio } & \multicolumn{6}{|c|}{ Negocios } \\
\hline & Don Juan & Estelí & Chaman & Cándida & Castillo & Sazón norteño \\
\hline Acceso WIFI & $0 \%$ & $0 \%$ & $0 \%$ & $8 \%$ & $1 \%$ & $9 \%$ \\
\hline Estacionamiento & $0 \%$ & $3 \%$ & $5 \%$ & $7 \%$ & $1 \%$ & $11 \%$ \\
\hline Área verde & $4 \%$ & $0 \%$ & $0 \%$ & $0 \%$ & $3 \%$ & $2 \%$ \\
\hline Acceso a personas con discapacidad & $2 \%$ & $3 \%$ & $2 \%$ & $4 \%$ & $7 \%$ & $3 \%$ \\
\hline Pasillos amplios para circulación & $9 \%$ & $11 \%$ & $12 \%$ & $10 \%$ & $8 \%$ & $6 \%$ \\
\hline Ventilación en todo el local & $12 \%$ & $12 \%$ & $7 \%$ & $6 \%$ & $9 \%$ & $9 \%$ \\
\hline Iluminación natural & $12 \%$ & $11 \%$ & $17 \%$ & $6 \%$ & $9 \%$ & $10 \%$ \\
\hline Iluminación artificial & $5 \%$ & $9 \%$ & $7 \%$ & $6 \%$ & $9 \%$ & $5 \%$ \\
\hline Higiene del local & $14 \%$ & $18 \%$ & $17 \%$ & $13 \%$ & $11 \%$ & $9 \%$ \\
\hline Mesas con suficiente espacio & $9 \%$ & $14 \%$ & $9 \%$ & $9 \%$ & $11 \%$ & $9 \%$ \\
\hline Mesas en buenas condiciones & $13 \%$ & $8 \%$ & $13 \%$ & $11 \%$ & $11 \%$ & $10 \%$ \\
\hline Atención en las mesas & $6 \%$ & $7 \%$ & $3 \%$ & $11 \%$ & $11 \%$ & $6 \%$ \\
\hline Pago con tarjetas & $8 \%$ & $5 \%$ & $7 \%$ & $1 \%$ & $5 \%$ & $6 \%$ \\
\hline Pago con moneda extranjera & $6 \%$ & $5 \%$ & $1 \%$ & $7 \%$ & $6 \%$ & $5 \%$ \\
\hline
\end{tabular}

Fuente: Elaboración propia

En la tabla $\mathrm{N}^{\circ} 4$, se observan que de las catorce condiciones de comodidad resaltadas por el cliente en los seis establecimientos, los que más sobresalen están: pasillos amplios para circulación, ventilación en todo el local, iluminación natural, higiene en todo el local, mesas con suficiente espacio, mesas en buenas condiciones. Es importante indicar que las condiciones varían en cada local, sin dejar de señalar que los seis establecimientos brindan comodidad a los clientes, ya que las encuestas aplicadas fueron diferenciadas en cantidad en cada establecimiento, debido a la cantidad de clientes que atienden diariamente.

El acceso a wifi solo lo brinda el buffet Sazón norteño y el comedor Cándida, la atención en las mesas la da el Castillo's buffet y el comedor Cándida. 
El buffet don Juan cuenta con 12 condiciones y no cuenta con acceso wifi y estacionamiento, el buffet Estelí cuenta con 12 condiciones, faltándole el área verde y acceso wifi, el buffet Chaman cuenta con 12 condiciones, no cuenta con acceso wifi y área verde, el comedor Cándida cuenta con 13 condiciones, faltándole área verde, el Castillo's buffet cuenta con las 14 condiciones, teniendo en mejores porcentajes el acceso wifi y estacionamiento, el buffet Sazón norteño cuenta con las 14 condiciones y presenta en menores porcentajes el área verde y acceso para personas con discapacidad.

Para aceptar tal afirmación de comodidad se realizó observación en los seis establecimientos, confirmando la información dada por el cliente, la cual se presenta en el siguiente gráfico:

Gráfico 3: Condiciones de comodidad en los locales

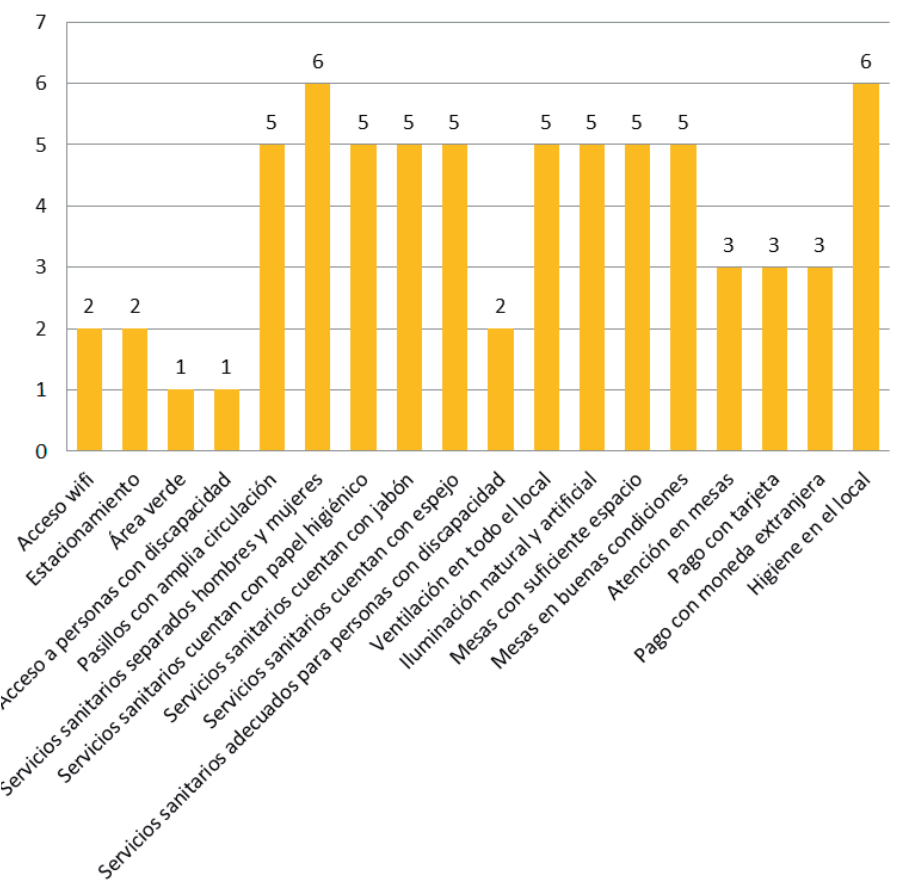

Fuente: Elaboración propia
En el gráfico número 3, las condiciones de comodidad identificadas en la guía de observación arroja dieciocho elementos con respecto a los que cliente destacó en la encuesta; en los seis locales sobresale la higiene en todo el local y los servicios sanitarios separados para hombres y mujeres.

Otros elementos son: pasillos amplios para circulación, los servicios sanitarios cuentan con papel higiénico, jabón y espejo, ventilación en todo el local, iluminación natural y artificial, mesas con suficiente espacio, mesas en buenas condiciones, ventilación en todo el local, iluminación natural y artificial, todos estos elementos en cinco de los seis locales respectivamente.

Los servicios sanitarios adecuados para personas con discapacidad, están en dos locales comedor Cándida y Castillo's buffet.

Con la descripción anterior se ratifica que los establecimientos cuentan con condiciones básicas que dan comodidad a los clientes. Otro aspecto destacado en la observación y no reflejado en el gráfico es que los locales cuentan con señalización de ruta de evacuación.

A continuación se analizará la aplicación de las estrategias que utilizan los propietarios con respecto a la teoría que plantea la autora Weinberger Villarán, es pertinente realizar un resumen de cuál es el factor de las estrategias competitivas que ponen en práctica los propietarios de los establecimientos, a continuación los datos: 
Tabla No 5: Resumen de implementación de estrategias competitivas por los propietarios

\begin{tabular}{|c|c|c|c|c|c|c|}
\hline $\begin{array}{l}\text { Estrategia planteadas por la } \\
\text { autora Karen Weinberger }\end{array}$ & $\begin{array}{c}\text { Buffet don } \\
\text { Juan }\end{array}$ & $\begin{array}{c}\text { Castillo's } \\
\text { buffet }\end{array}$ & $\begin{array}{c}\text { Buffet } \\
\text { Chaman }\end{array}$ & $\begin{array}{l}\text { Comedor } \\
\text { Cándida } \\
\end{array}$ & $\begin{array}{l}\text { Buffet } \\
\text { Estelí }\end{array}$ & $\begin{array}{c}\text { Buffet } \\
\text { Sazón norteño } \\
\end{array}$ \\
\hline \multicolumn{7}{|c|}{ Liderazgo en costos } \\
\hline Factores para fijar precios & $\sqrt{ }$ & $\sqrt{ }$ & $\sqrt{ }$ & $\sqrt{ }$ & $\sqrt{ }$ & $\sqrt{ }$ \\
\hline Relación con los proveedores & $\sqrt{ }$ & $\sqrt{ }$ & $\sqrt{ }$ & $\sqrt{ }$ & $\sqrt{ }$ & $\sqrt{ }$ \\
\hline \multicolumn{7}{|c|}{ Diferenciación } \\
\hline Productos que entreguen valor & $\sqrt{ }$ & $\sqrt{ }$ & $\sqrt{ }$ & $\sqrt{ }$ & $\sqrt{ }$ & $\sqrt{ }$ \\
\hline Rotación del menú & $\sqrt{ }$ & $\sqrt{ }$ & $\sqrt{ }$ & $\sqrt{ }$ & $\sqrt{ }$ & $\sqrt{ }$ \\
\hline Innovación & $\mathrm{X}$ & $\mathrm{X}$ & $\mathrm{X}$ & $X$ & $\mathrm{X}$ & $X$ \\
\hline \multicolumn{7}{|c|}{ Enfoque } \\
\hline Porción del mercado & $\sqrt{ }$ & $\sqrt{ }$ & $\sqrt{ }$ & $\sqrt{ }$ & $\sqrt{ }$ & $\sqrt{ }$ \\
\hline Tipo de clientes & $\sqrt{ }$ & $\sqrt{ }$ & $\sqrt{ }$ & $\sqrt{ }$ & $\sqrt{ }$ & $\sqrt{ }$ \\
\hline Lealtad & $\sqrt{ }$ & $\sqrt{ }$ & $\sqrt{ }$ & $\sqrt{ }$ & $\sqrt{ }$ & $\sqrt{ }$ \\
\hline $\begin{array}{l}\text { Capacidades y recursos necesarios } \\
\text { (desde cuando es cliente, años } \\
\text { operación, cantidad de trabajadores) }\end{array}$ & $\sqrt{ }$ & $\sqrt{ }$ & $\sqrt{ }$ & $\sqrt{ }$ & $\sqrt{ }$ & $\sqrt{ }$ \\
\hline $\begin{array}{l}\text { Transmisión de estrategia a los } \\
\text { trabajadores }\end{array}$ & $\sqrt{ }$ & $\sqrt{ }$ & $\sqrt{ }$ & $\sqrt{ }$ & $\sqrt{ }$ & $\sqrt{ }$ \\
\hline
\end{tabular}

Fuente: Elaboración propia

En la tabla número 5, se puede observar que los propietarios ponen en práctica nueve de los diez factores de las tres estrategias competitivas que plantea la autora Weinberger, a excepción de la innovación.
Según las encuestas proporcionadas a los clientes que visitan los establecimientos indicaron que los propietarios de los seis establecimientos utilizan elementos del marketing de servicio.

Objetivo número 2: Describir los elementos del marketing de servicio que utilizan las MIPYMES del sector servicios de comida buffet en la ciudad de Estelí.

Tabla $\mathbf{N}^{\circ}$ 6: Elementos del marketing ponen en practica en el negocio

\begin{tabular}{lrrrrrr}
\hline \multirow{2}{*}{8 P's de marketing de servicio } & \multicolumn{5}{c}{ Negocios } \\
\cline { 2 - 6 } & Sazón Norteño & Don Juan & Chaman & Estelí & Cándida & Castillo \\
\hline Satisfacción por el producto & $15 \%$ & $15 \%$ & $16 \%$ & $16 \%$ & $14 \%$ & $15 \%$ \\
Rapidez y comodidad del lugar & $16 \%$ & $12 \%$ & $15 \%$ & $22 \%$ & $14 \%$ & $14 \%$ \\
El precio pagado por el servicio & $12 \%$ & $15 \%$ & $15 \%$ & $16 \%$ & $10 \%$ & $14 \%$ \\
Promociones y publicidad & $2 \%$ & $1 \%$ & $1 \%$ & $0 \%$ & $1 \%$ & $3 \%$ \\
Proceso de entrega del producto & $14 \%$ & $14 \%$ & $14 \%$ & $15 \%$ & $15 \%$ & $13 \%$ \\
Apariencia del lugar & $16 \%$ & $15 \%$ & $16 \%$ & $0 \%$ & $15 \%$ & $14 \%$ \\
El personal establece comunicación & $11 \%$ & $14 \%$ & $8 \%$ & $11 \%$ & $15 \%$ & $13 \%$ \\
con el cliente & & & & & & $17 \%$ \\
Agilidad y calidad del servicio & $14 \%$ & $15 \%$ & $17 \%$ & $21 \%$ & $14 \%$ \\
\hline
\end{tabular}

Fuente: Elaboración propia 
En la tabla 6, los elementos del marketing de servicio que más sobresalen en los datos según las encuestas aplicadas a los clientes están: Satisfacción por el producto es decir elementos del producto, rapidez y comodidad del lugar es decir lugar y tiempo, precio pagado por el servicio es decir precio y otros costos para el usuario, proceso de entrega del producto es decir proceso, apariencia del lugar es decir entorno físico, el personal establece comunicación con el cliente es decir personal y agilidad y calidad del servicio es decir productividad y calidad. De los ocho elementos del marketing de servicio solo un elemento no fue tan seleccionado por los clientes como es promociones y publicidad es decir promoción y educación, hecho marcado en los seis establecimientos, sin embargo, es importante analizar que en un porcentaje del $3 \%$ el Castillo's buffet y el 2\% en el buffet Sazón norteño indicaron que en los establecimientos se utiliza este elemento promociones y publicidad.

Al consultarle al propietario en entrevista sobre los elementos del marketing de servicio que ha utilizado expreso lo siguiente:

Tabla $\mathbf{N}^{\circ}$ 7: Elementos del marketing utilizados por propietarios

\begin{tabular}{ll}
\hline $\begin{array}{c}\text { Nombre del } \\
\text { establecimiento }\end{array}$ & \multicolumn{1}{c}{ Comentarios según entrevista } \\
\hline Buffet Don Juan & $\begin{array}{l}\text { "Si, más que todo la publicidad y } \\
\text { precios". (Alvares, 2016) }\end{array}$ \\
"Si, pero no siempre”. (Sevilla, \\
Castillo's buffet & $\begin{array}{l}\text { 2016) } \\
\text { Buffet Chaman }\end{array}$ \\
$\begin{array}{l}\text { "Si, la publicidad” (Reyes, 2016) } \\
\text { "Variedad en menú". (Gutiérrez, }\end{array}$ \\
$\begin{array}{l}\text { Buffet Estelín } \\
\text { Buffet Sazón } \\
\text { norteño }\end{array}$ & "Si. El Facebook". (Orozco, 2016) \\
\hline
\end{tabular}

Fuente: Elaboración propia

Los seis propietarios afirmaron utilizar elementos del marketing de servicio, los más utilizados son: publicidad, precio, variedad en el menú. Más sin embargo, el propietario del Castillo's buffet indicó que no utilizar siempre el marketing.

Las 8 p's que enuncia el autor Lovelock son: elementos del producto, lugar y tiempo, precio y otros costos para el usuario, promoción y educación, entorno físico, proceso, personal y productividad y calidad.

A continuación se analizan algunos elementos del marketing que utilizan los propietarios de los seis establecimientos.

En cuanto a los elementos del producto se consultó a los propietarios si ofertan la variedad de productos:

Tabla $\mathbf{N}^{\circ}$ 8: Variedad de productos

\begin{tabular}{|c|c|}
\hline $\begin{array}{c}\text { Nombre del } \\
\text { establecimiento }\end{array}$ & Comentarios según entrevista \\
\hline Buffet Don Juan & $\begin{array}{l}\text { "Si, siempre tratamos de ofrecer un } \\
\text { menú diferente". (Alvares, 2016) }\end{array}$ \\
\hline Castillo's buffet & $\begin{array}{l}\text { "Si, claro si, tenemos más de } 25 \\
\text { opciones de carnes y } 15 \text { opciones de } \\
\text { ensaladas". (Sevilla, 2016) }\end{array}$ \\
\hline Buffet Chaman & $\begin{array}{l}\text { "Si, tenemos } 6 \text { tipos de carnes } \\
\text { diarias, } 5 \text { tipos de ensaladas, pastas, } \\
\text { guisos, } 4 \text { tipo de bastimento y } 3 \text { de } \\
\text { refresco". (Reyes, 2016) }\end{array}$ \\
\hline Comedor Cándida & $\begin{array}{l}\text { "Si, } 10 \text { a } 12 \text { platos en comida } \\
\text { corriente y variada de ensaladas". } \\
\text { (Gutiérrez, 2016) }\end{array}$ \\
\hline Buffet Estelí & $\begin{array}{l}\text { "Si. } 5 \text { variedades de carnes, } 3 \text { de } \\
\text { frescos y gaseosas, } 4 \text { de ensaladas } \\
\text { y } 5 \text { tipos de bastimento." (Orozco, } \\
\text { 2016) }\end{array}$ \\
\hline $\begin{array}{l}\text { Buffet Sazón } \\
\text { norteño }\end{array}$ & $\begin{array}{l}\text { "Si, ofrecemos } 12 \text { tipo de carnes, } 4 \\
\text { tipos de ensaladas". (Ruiz, 2016) }\end{array}$ \\
\hline
\end{tabular}

Fuente: Elaboración propia

En los seis establecimientos los propietarios ofrecen variedad de productos, para ofrecer un menú diferente al cliente, es considerable variedad de carnes, ensaladas, bastimentos, guisos y refrescos naturales. 
De acuerdo a la observación realizada en los establecimientos la variedad de productos difiere en los locales, a continuación los resultados:

\section{Gráfico 4:}

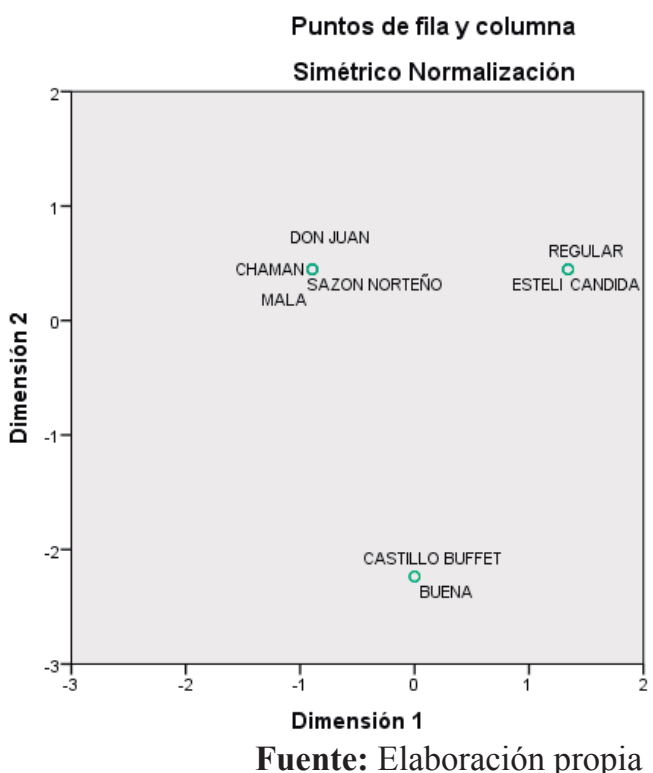
NOMBRE DEL
ESTABLECIMENTO
OVARIEDAD DE PRODUCTOS

Igualmente lo expresado en la entrevista por los dueños de los establecimientos se confirma haciendo uso de un análisis de correspondencia utilizando el SPSS aplicándolo a los resultados de la guía de observación (ver gráfico 4) donde es claro que la variedad de productos es estimada como buena solamente en Castillo Buffet, no así en Don Juan, Chaman y Sazón Norteño y quien está en el medio esta buffet Estelí y comedor Cándida.

De esta manera se confirma nuevamente que Castillo Buffet es uno de los establecimientos que está en la preferencia de los clientes por la variedad de productos que oferta.

En cuanto a la variedad de productos es importante que se oferte una gama de productos y amplitud de productos al cliente para que este tenga la opción de decisión entre varias opciones. Para los propietarios es fundamental que se realice una variedad de lo que ofertan atendiendo la temporada en cuanto a la oferta que les hacen los proveedores pero sobre todo de acuerdo a lo que más demandan los clientes. De hecho la variedad les puede generar una ventaja competitiva.

La teoría del primer elemento del marketing de servicio (elementos del producto) expone que los productos de servicio constituyen el núcleo de la estrategia de marketing de una empresa. Los productos de servicios consisten en un bien fundamental, que responde a una necesidad primaria del cliente, y en un conjunto de elementos del servicio complementario. (Lovelock \& Wirtz, 2009)

En cuanto al segundo elementos lugar y tiempo: la exhibición de los productos en los seis establecimientos es igual, a continuación datos según entrevista:

Tabla Nº: Exhibición de los productos

Nombre del
establecimiento $\quad$ Comentarios según entrevista

\begin{tabular}{|c|c|}
\hline Buffet Don Juan & $\begin{array}{l}\text { "A través del buffet o se le conoce } \\
\text { como exhibidor de comida en baño } \\
\text { María y las ensaladas en el exhibidor } \\
\text { en frío". (Alvares, 2016) }\end{array}$ \\
\hline Castillo's buffet & “En baño María”. (Sevilla, 2016) \\
\hline Buffet Chaman & $\begin{array}{l}\text { "Tradicional baño } \quad \text { María". } \\
\text { (Reyes, 2016) }\end{array}$ \\
\hline Comedor Cándida & $\begin{array}{l}\text { "Baño María en frio y caliente". } \\
\text { (Gutiérrez, 2016) }\end{array}$ \\
\hline Buffet Estelí & “Baño María”. (Orozco, 2016) \\
\hline $\begin{array}{l}\text { Buffet Sazón } \\
\text { norteño }\end{array}$ & “En baño María”. (Ruiz, 2016) \\
\hline
\end{tabular}

Fuente: Elaboración propia

La exhibición de los productos o alimentos es realizada a través de las cocina baño María, en caliente y en frio como es en el caso de las ensaladas, este dato se confirmó al realizar la observación directa en los establecimientos mientras la investigadora aplicaba encuestas a los clientes.

El cuarto elemento es promoción y educación, se consultó a los clientes si identifican que en los establecimientos hay promociones, a continuación datos según encuesta: 
Gráfico 5: El buffet ofrece promociones

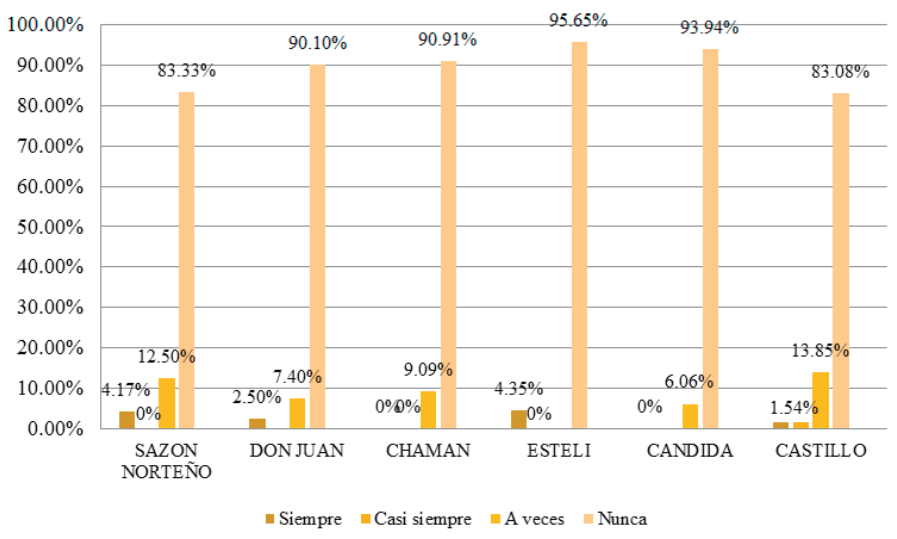

Fuente: Elaboración propia

En el gráfico número 5 , los clientes indican que en los seis establecimientos nunca hay promociones. En cuatro establecimientos se refleja que a veces hay promociones, lo cual no es muy significativo, el porcentaje es de $13.85 \%$ a $6.06 \%$ y en menor porcentajes los clientes indican que siempre hay promociones en porcentajes de $4.35 \%$ a $1.54 \%$, lo cual no es muy significativo.

También se les consultó a los propietarios si realizan promoción en sus locales y los medios que utiliza para dar a conocer sus establecimientos, a continuación datos:

Tabla $N^{\circ}$ 10: Promoción en los locales y medios utilizados

\begin{tabular}{cc}
\hline $\begin{array}{c}\text { Nombre del } \\
\text { establecimiento }\end{array}$ & Comentarios según entrevista \\
\hline
\end{tabular}

"No se realizan promociones. Se utiliza publicidad cada tres meses en revistas (Revista selecciones y el Esteliano), apoyando a equipos Buffet Don Juan deportivos de fútbol, perifoneo y por la radio (radio stereo única), es necesario utilizar la publicidad para crecer y no dejar el nombre del negocio dormido". (Alvares, 2016)
"No realizamos promoción en descuentos, la mejor publicidad que tenemos es la de los clientes, Castillo's buffet pero si utilizamos publicidad en radio permanente, hay mucha gente que no saben de la existencia de nuestro negocio y así captamos clientes nuevos". (Sevilla, 2016)

"No se realizan promociones, se utiliza la radio mensualmente, las volantes y la revista de Gira cada tres meses, es importante mantener la publicidad para atraer al cliente". (Reyes, 2016)

"No se realizan promociones, se utiliza publicidad en perifoneo $\mathrm{y}$

Comedor Cándida la radio, lo hacemos constante, sin publicidad no hay nada." (Gutiérrez, 2016)

"No, promociones no hacemos, me falta hacer promociones. $\mathrm{Si}$ hemos utilizado los medios de Buffet Estelí comunicación para hacer publicidad de nuestro local a través de la Radio, por internet y el negocio tiene su página de Facebook, así damos a conocer el negocio". (Orozco, 2016) "No se realizan promociones, no Buffet Sazón norteño uso ningún método publicitario solo la recomendaciones de nuestros clientes". (Ruiz, 2016)

Fuente: Elaboración propia

En los seis establecimientos no se realizan promociones sobre los productos. En los primeros cinco establecimientos han utilizado medios publicitarios, consideran importante recordarle al cliente su ubicación y su nombre, en el caso de Castillo's buffet y buffet Sazón norteño consideran importante la publicidad que les da el cliente que los visita, así recomiendan el local, en el buffet Sazón norteño la propietaria indica que no utiliza publicidad y que solo espera que el cliente que la visita le de publicidad.

Las promociones y regalías son acciones de estrategia de servicios, un 50\% afirma que si realiza regalías (datos 
en análisis primer objetivo, estrategias competitivas página 95 ) y no realiza promociones a sus clientes, por lo que se puede rescatar la idea de hacer promociones y regalías a los clientes que se estimen son los más frecuentes o los que se destacan por fidelidad a lo largo de la permanencia del negocio en el mercado, esto le dará satisfacción a los clientes y un sentido de gratitud y pertenencia.

El quinto elemento del marketing de servicio es el proceso, se consultó en encuesta a los clientes sobre el tiempo en que tardan en ser atendidos, a continuación los datos:

Gráfico 6: Tiempo en que tarda en ser atendido

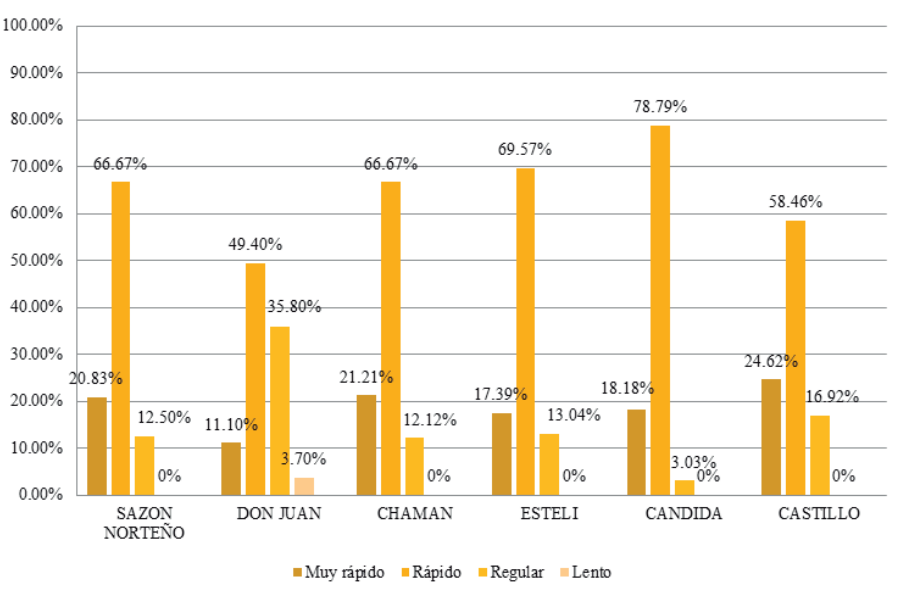

Fuente: Elaboración propia

En los datos del gráfico número 6, se puede observar que en los seis establecimientos el tiempo que tardan ser atendidos los clientes es rápido, en el buffet Sazón norteño tiene un porcentaje de $66.67 \%$, en el buffet don Juan el porcentaje es de $49.40 \%$, en el buffet Chaman el porcentaje es de $66.67 \%$, en el buffet Estelí el porcentaje es de $69.57 \%$, en el comedor Cándida el porcentaje es $78.79 \%$ y el Castillo's buffet el porcentaje es de $58.46 \%$. Es importante destacar que el tiempo de atención es muy importante para el cliente y por lo tanto en el buffet don Juan y en el Castillo's buffet deben de prestar atención a este aspecto, debido a las bajas porcentuales con respecto a los otros cuatro locales.
Con respecto a la guía de observación en el tiempo de atención se obtuvieron los siguientes resultados:

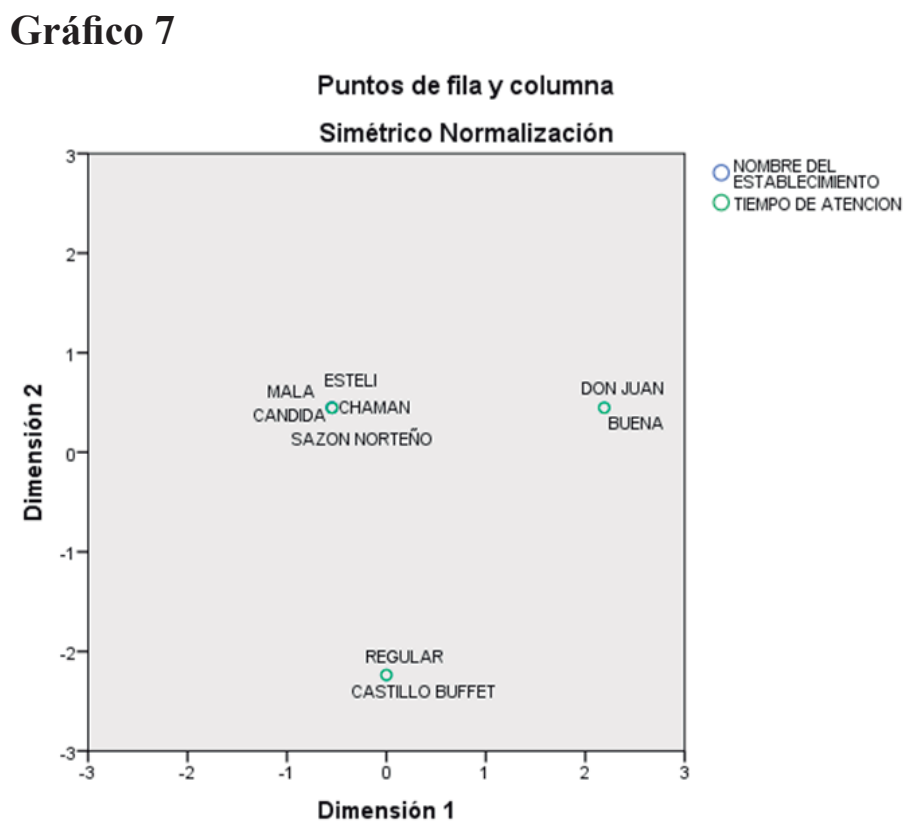

Fuente: Elaboración propia

Al realizar un análisis de correspondencia entre las variables nombre del establecimiento y el tiempo de espera para ser atendido los resultados en el Castillo's buffet donde el tiempo de atención es regular en los demás establecimientos mala y en este aspecto se destaca el buffet don Juan donde se pudo constatar vía observación que el tiempo de espera para ser atendido está ubicado en la categoría de buena, es decir que es bastante aceptable.

Por tanto el Buffet Don Juan puede tomar cierta ventaja de esta variable para que sus clientes comenten con otros posibles clientes que la atención es ágil y el tiempo que es apremiante a la hora del almuerzo en ese local no es un problema.

Después de haber realizado el análisis de la implementación de las 8 p's del marketing de servicio que plantea los autores Christopher Lovelock y Jochen Wirtz, es pertinente realizar un resumen de cuál es el elemento del marketing de servicio que ponen en práctica los propietarios de los establecimientos, a continuación los datos: 
Tabla $\mathbf{N}^{\mathbf{1}} 11$ Resumen de implementación de 8 P's del marketing de servicio

\begin{tabular}{|c|c|c|c|c|c|c|}
\hline $\begin{array}{c}\text { Estrategia planteadas por la autora } \\
\text { Karen Weinberger } \\
\end{array}$ & $\begin{array}{c}\text { Buffet don } \\
\text { Juan }\end{array}$ & $\begin{array}{c}\text { Castillo's } \\
\text { buffet }\end{array}$ & $\begin{array}{c}\text { Buffet } \\
\text { Chaman }\end{array}$ & $\begin{array}{l}\text { Comedor } \\
\text { Cándida }\end{array}$ & $\begin{array}{l}\text { Buffet } \\
\text { Estelí }\end{array}$ & $\begin{array}{c}\text { Buffet } \\
\text { Sazón norteño } \\
\end{array}$ \\
\hline \multicolumn{7}{|c|}{ Elementos del producto } \\
\hline Variedad de productos & $\sqrt{ }$ & $\sqrt{ }$ & $\sqrt{ }$ & $\sqrt{ }$ & $\sqrt{ }$ & $\sqrt{ }$ \\
\hline \multicolumn{7}{|c|}{ Lugar y tiempo } \\
\hline Exhibición productos & $\sqrt{ }$ & $\sqrt{ }$ & $\sqrt{ }$ & $\sqrt{ }$ & $\sqrt{ }$ & $\sqrt{ }$ \\
\hline Rapidez al momento de entrega & $\sqrt{ }$ & $\sqrt{ }$ & $X$ & $\sqrt{ }$ & $\sqrt{ }$ & $\sqrt{ }$ \\
\hline \multicolumn{7}{|c|}{ Precios y otros costos para el usuario } \\
\hline Precio del producto & $\sqrt{ }$ & $\sqrt{ }$ & $\sqrt{ }$ & $\sqrt{ }$ & $\sqrt{ }$ & $\sqrt{ }$ \\
\hline \multicolumn{7}{|c|}{ Promoción y educación } \\
\hline Promoción & $X$ & $X$ & $X$ & $X$ & $X$ & $X$ \\
\hline Medios publicitarios & $\sqrt{ }$ & $\sqrt{ }$ & $\sqrt{ }$ & $\sqrt{ }$ & $\sqrt{ }$ & $X$ \\
\hline \multicolumn{7}{|c|}{ Procesos } \\
\hline Proceso de atención al cliente & $\sqrt{ }$ & $\sqrt{ }$ & $\sqrt{ }$ & $\sqrt{ }$ & $\sqrt{ }$ & $\sqrt{ }$ \\
\hline Tiempo de atención & $\sqrt{ }$ & $\sqrt{ }$ & $X$ & $\sqrt{ }$ & $\sqrt{ }$ & $\sqrt{ }$ \\
\hline \multicolumn{7}{|c|}{ Entorno Físico } \\
\hline Condiciones de los establecimientos & $\sqrt{ }$ & $\sqrt{ }$ & $\sqrt{ }$ & $\sqrt{ }$ & $\sqrt{ }$ & $\sqrt{ }$ \\
\hline Accesibilidad & $\sqrt{ }$ & $\sqrt{ }$ & $\sqrt{ }$ & $\sqrt{ }$ & $\sqrt{ }$ & $\sqrt{ }$ \\
\hline Equipos & $\sqrt{ }$ & $\sqrt{ }$ & $\sqrt{ }$ & $\sqrt{ }$ & $\sqrt{ }$ & $\sqrt{ }$ \\
\hline Apariencia del personal & $\sqrt{ }$ & $\sqrt{ }$ & $\sqrt{ }$ & $\sqrt{ }$ & $\sqrt{ }$ & $\sqrt{ }$ \\
\hline Señales visibles en los locales & $\sqrt{ }$ & $\sqrt{ }$ & $\sqrt{ }$ & $\sqrt{ }$ & $\sqrt{ }$ & $\sqrt{ }$ \\
\hline \multicolumn{7}{|c|}{ Personal } \\
\hline Interacción directa con el cliente & $\sqrt{ }$ & $\sqrt{ }$ & $\sqrt{ }$ & $\sqrt{ }$ & $\sqrt{ }$ & $\sqrt{ }$ \\
\hline \multicolumn{7}{|c|}{ Productividad y calidad } \\
\hline Reducción de costos & $\sqrt{ }$ & $\sqrt{ }$ & $X$ & $\sqrt{ }$ & $\sqrt{ }$ & $\sqrt{ }$ \\
\hline Calidad del producto & $\sqrt{ }$ & $\sqrt{ }$ & $\sqrt{ }$ & $\sqrt{ }$ & $\sqrt{ }$ & $\sqrt{ }$ \\
\hline
\end{tabular}

Fuente: Elaboración propia

Con respecto a la tabla número 31 , se puede observar que en los seis establecimientos los propietarios implementan 7 elementos de las 8 p's del elementos del marketing de servicio, a excepción del cuarto elemento promoción y educación, los propietarios indicaron no realizar promociones en los locales, y con respecto a la utilización de los medios publicitarios el único establecimiento que no hace no utiliza es el buffet Sazón norteño. En relación al octavo elemento productividad y calidad, el propietario del buffet
Chaman indicio que no reduce costos para no sacrificar la calidad del producto final.

Objetivo número 3: Identificar la competencia que hay en las MIPYME del sector servicio de comida buffet en la ciudad de Estelí

En entrevista, se consultó a los propietarios si tienen conocimiento de la competencia en el sector de comida buffet, a continuación los datos: 
Tabla $N^{\circ}$ 12: Conocimiento de competencia en el sector

Nombre del
establecimiento Comentarios según entrevista

"Si, tengo varios competidores

Buffet Don Juan se podría decir que los que están alrededor del negocio". (Alvares, 2016)

"Sé que hay muchos competidores pero para nosotros la competencia somos nosotros mismos." (Sevilla, 2016)

"Si, buffet Castillo y el buffet don

Buffet Chaman
" $\mathrm{Si}$, son muchos, demasiados diría yo.

Comedor Cándida Sabor Nicaragüense, buffet Castillo, Quesito N 1 y 2". (Gutiérrez, 2016) "Si los conozco. El Chaman, el Buffet Estelí buffet Castillo y buffet don Juan". (Orozco, 2016)

Buffet Sazón "Si, el Sabor Nicaragüense que es el norteño que queda más cerca." (Ruiz, 2016)

Fuente: Elaboración propia

La autora Nelly Cárdenas Dávila plantea nueve factores determinantes de la competitividad a nivel de empresa, es pertinente realizar un resumen de los factores para reflejar los que ponen en práctica los propietarios de los establecimientos, a continuación los datos:

Tabla $N^{\mathbf{0}}$ 13: Resumen de implementación de factores determinantes de competitividad

\begin{tabular}{|c|c|c|c|c|c|c|}
\hline $\begin{array}{c}9 \text { Factores determinantes de la } \\
\text { competitividad planteados por la autora } \\
\text { Nelly Cárdenas Dávila }\end{array}$ & $\begin{array}{c}\text { Buffet don } \\
\text { Juan }\end{array}$ & $\begin{array}{c}\text { Castillo's } \\
\text { buffet }\end{array}$ & $\begin{array}{l}\text { Buffet } \\
\text { Cha- } \\
\text { man }\end{array}$ & $\begin{array}{l}\text { Comedor } \\
\text { Cándida }\end{array}$ & $\begin{array}{l}\text { Buffet } \\
\text { Estelí }\end{array}$ & $\begin{array}{c}\text { Buffet } \\
\text { Sazón norteño }\end{array}$ \\
\hline \multicolumn{7}{|c|}{ Capacidades directivas } \\
\hline Capacidades de dirección & $\sqrt{ }$ & $\sqrt{ }$ & $\sqrt{ }$ & $\sqrt{ }$ & $\sqrt{ }$ & $\sqrt{ }$ \\
\hline Liderazgo & $\sqrt{ }$ & $\sqrt{ }$ & $\sqrt{ }$ & $\sqrt{ }$ & $\sqrt{ }$ & $\sqrt{ }$ \\
\hline Implementación del proceso administrativo & $\sqrt{ }$ & $\sqrt{ }$ & $\sqrt{ }$ & $\sqrt{ }$ & $\sqrt{ }$ & $\sqrt{ }$ \\
\hline Toma de decisiones & $\sqrt{ }$ & $\sqrt{ }$ & $\sqrt{ }$ & $\sqrt{ }$ & $\sqrt{ }$ & $\sqrt{ }$ \\
\hline \multicolumn{7}{|c|}{ Producción o prestación servicios-diferenciación } \\
\hline Calidad de los productos & $\sqrt{ }$ & $\sqrt{ }$ & $\sqrt{ }$ & $\sqrt{ }$ & $\sqrt{ }$ & $\sqrt{ }$ \\
\hline Diferenciación de productos & $\sqrt{ }$ & $\sqrt{ }$ & $\sqrt{ }$ & $\sqrt{ }$ & $\sqrt{ }$ & $\sqrt{ }$ \\
\hline Atención brindada al cliente & $\sqrt{ }$ & $\sqrt{ }$ & $\sqrt{ }$ & $\sqrt{ }$ & $\sqrt{ }$ & $\sqrt{ }$ \\
\hline \multicolumn{7}{|c|}{ Ventajas competitivas (costo-calidad) } \\
\hline Costo del producto & $\sqrt{ }$ & $\sqrt{ }$ & $\sqrt{ }$ & $\sqrt{ }$ & $\sqrt{ }$ & $\sqrt{ }$ \\
\hline $\begin{array}{l}\text { Calidad (oferta de productos únicos que } \\
\text { generen valor) }\end{array}$ & $\sqrt{ }$ & $\sqrt{ }$ & $\sqrt{ }$ & $\sqrt{ }$ & $\sqrt{ }$ & $\sqrt{ }$ \\
\hline
\end{tabular}

\begin{tabular}{|c|c|c|c|c|c|c|}
\hline \multicolumn{7}{|c|}{ Recursos tecnológicos } \\
\hline Equipos & $\sqrt{ }$ & $\sqrt{ }$ & $\sqrt{ }$ & $\sqrt{ }$ & $\sqrt{ }$ & $\sqrt{ }$ \\
\hline \multicolumn{7}{|c|}{ Innovación } \\
\hline Innovación de productos & $X$ & $X$ & $X$ & $X$ & $X$ & $X$ \\
\hline \multicolumn{7}{|c|}{ Relaciones comerciales } \\
\hline Tipos de clientes & $\sqrt{ }$ & $\sqrt{ }$ & $\sqrt{ }$ & $\sqrt{ }$ & $\sqrt{ }$ & $\sqrt{ }$ \\
\hline Fidelidad del cliente & $\sqrt{ }$ & $\sqrt{ }$ & $\sqrt{ }$ & $\sqrt{ }$ & $\sqrt{ }$ & $\sqrt{ }$ \\
\hline Campañas para fortalecer marca & $\sqrt{ }$ & $\sqrt{ }$ & $\sqrt{ }$ & $\sqrt{ }$ & $\sqrt{ }$ & $\sqrt{ }$ \\
\hline
\end{tabular}

Campañas para fortalecer marca

Recursos humanos-capacitación laboral

Proceso de contratación de personal

$\begin{array}{r}\begin{array}{c}\text { Buffet d } \\ \text { Juan }\end{array} \\ \text { Capa } \\ \text { Rón o prestac } \\ \hline \text { Recur }\end{array}$


Capacitaciones

Retención laboral

\begin{tabular}{lccccccc}
\hline \multicolumn{7}{c}{ Recursos financieros } \\
\hline Inversión en los negocios & $\sqrt{ }$ & $\sqrt{ }$ & $\sqrt{ }$ & $\sqrt{ }$ & $\sqrt{ }$ & $\sqrt{ }$ \\
Planificación financiera & $\sqrt{ }$ & $\sqrt{ }$ & $\sqrt{ }$ & $\sqrt{ }$ & $\sqrt{ }$ & $\sqrt{ }$ \\
\hline \multicolumn{7}{c}{ Cultura } \\
\hline $\begin{array}{l}\text { Relación con el personal, transmisión de } \\
\text { costumbres, hábitos y valores }\end{array}$ & $\sqrt{ }$ & $\sqrt{ }$ & $\sqrt{ }$ & $\sqrt{ }$ & $\sqrt{ }$ \\
\hline
\end{tabular}

Fuente: Elaboración propia

Con respecto a la tabla número 37 , se puede observar que en los seis establecimientos los propietarios implementan 8 de los 9 factores determinantes de la competitividad, a excepción del quinto factor; innovación, los propietarios indicaron que ellos varían el menú diariamente y semanalmente, lo cual no es propiamente innovar, la teoría plantea que el éxito de las empresas también se asocia al desarrollo de nuevos productos.

Con respecto al séptimo factor recursos humanoscapacitación laboral el buffet Sazón norteño no cumple con el proceso de capacitación al personal, únicamente con el proceso de contratación.

Objetivo 4: Proponer estrategias competitivas que permitan a las MIPYEMES del sector servicios de comida buffet competir

\section{Estrategia $N^{\circ}$ 1: La diferenciación}

Objetivo: Lograr ser únicos o negocios con características tangibles e intangibles que les diferencien del resto de la competencia

\section{Acciones:}

1. Mejora en cuanto a las condiciones de infraestructura del negocio sobre todo lo relacionado a aquellas que representan o son de mayor importancia para los clientes: Estacionamiento, WIFI, acceso para personas con discapacidad, etc.

2. Capacitación al personal para que pueda brindar mayor atención en mesas.
3. Oferta de promociones del día o de la semana, de forma tal que el cliente se sienta identificado y apreciado, generando esto la comodidad y fidelidad del cliente con el negocio.

\section{Estrategia $N^{\circ}$ 2: Promoción del servicio}

Objetivo: Presentar de una manera agradable y novedosa la calidad del servicio y la variedad de los productos (menú)

Acciones:

1. Uso de publicidad radial y televisiva

2. Patrocinio de ligas deportivas

3. Uso de bonos de clientes a los primeros 50 clientes que ingresen durante la hora del almuerzo

4. Ubicar un buzón de sugerencias

5. Crear mesas con condiciones especiales para los clientes más asiduos o clientes fieles por más de dos años de visitar el negocio

6. Creación de combos promocionales para ofertar al menos una vez por semana

7. Crear la hora feliz para que los clientes puedan tomar de manera libre café o dulces después del almuerzo

\section{Estrategia $N^{\circ}$ 3: Estrategia de posicionamiento}

Objetivo: Lograr el posicionamiento en la mente de los clientes

Acciones:

1. Realización de un estudio de mercado que le 
es su mercado meta.

2. Definición de qué tipo de posicionamiento es el que desea implementar y a qué nivel, en el caso de los servicios puede ser una óptima combinación de la mezcla de mercadotecnia

3. Presentación ante los clientes de una imagen de compromiso con ellos

4. Identificación de una ventaja competitiva

5. Tener claro el tipo de posicionamiento que se quiere implementar si es de líder, seguidor, atacante o de nicho de mercado.

6. Posicionamiento de la gama de productos o platillos que ofrece basándose en la calidad de los víveres que se utilizan para la elaboración de los mismos

7. Posicionamiento en base a la estructura de precios accesibles en relación a la competencia

8. Posicionamiento en función de mejorar lo que ya ofrece la competencia, esto puede ser a través de regalías, atención esmerada

\section{CONCLUSIONES}

Al analizar la opinión de los propietarios de los seis establecimientos en estudio, la de los clientes que visitan estos locales y las observaciones realizadas, se llegó a las siguientes conclusiones:

Los propietarios estiman utilizar estrategias, propiamente no se están implementando, sino que son acciones para crear condiciones de fidelidad en los clientes

Los seis propietarios afirmaron utilizar más ciertos elementos del marketing de servicio, publicidad, precio, variedad en el menú. El propietario Castillo's buffet indicó que no utiliza siempre el marketing. No existe un profesional de marketing que apoye a los propietarios en la promoción de sus locales

Los clientes indican que en los seis establecimientos de comida buffet hay competitividad entre sí, identificando a un líder en competitividad que sobre sale en cada uno, este es el Castillo's buffet.

En cuanto a la innovación, los propietarios confirman estar pendientes en ofertar variedad. La innovación de los productos no aplica para estos establecimientos, ya que no se ofertan nuevos productos a los clientes.

La calidad del producto los seis propietarios afirman ofertar productos de calidad, la que obtienen mediante diferentes procesos.

Las capacidades y los recursos necesarios para servir al nicho de manera efectiva (otro factor de la estrategia de enfoque): los propietarios tienen más de 1 año de establecimiento en el mercado hasta los 17 años. La cantidad de trabajadores se mantiene de 5 hasta los 15 , como en el caso del Castillo's buffet, tiene personal de cocina, meseros y personal de compra.

En la transmisión de las estrategias al personal, los seis propietarios lo hacen por medio de la comunicación directa al personal.

Las capacidades y los recursos necesarios para servir al nicho de manera efectiva (otro factor de la estrategia de enfoque): los propietarios tienen más de 1 año de establecimiento en el mercado hasta los 17 años. La cantidad de trabajadores se mantiene de 5 hasta los 15 , como en el caso del Castillo's buffet, tiene personal de cocina, meseros y personal de compra.

En la transmisión de las estrategias al personal, los seis propietarios lo hacen por medio de la comunicación directa al personal.

En el análisis del diamante de Porter, reflejó que en el poder de negociación con los proveedores, los propietarios obtienen los productos de los mismos proveedores a precios competitivos, negocia calidad de los vivires, entrega en el local, con opción de pago de crédito y de contado. 
En cuanto al poder de negociación con el cliente, los clientes exigen variedad de platillos, de calidad y sabor en tamaño de porciones, en ambientes y condiciones adecuadas.

Los productos sustitutos, hay variedad de oferta de productos, en comiderias, restaurantes y kioskos cercanas a lo locales, a precios bajos.

La rivalidad del sector/ poder de negociación de los competidores es oportuno crear alianzas para los dueños de los locales de comida buffet para beneficio de todos, precio de los platillos ajustados y acorde a los precios de los otros buffet y una oferta de variedad de menú, comodidad y condiciones modernas en los locales y de los servicios higiénicos, precios bajos, calidad de atención, ambiente y calidad de mobiliario en el local, oferta de regalías por compras en grupos.

La amenaza de nuevos competidores, la apertura de locales cercanos a los buffet y competidores usan economías de escala generando precio bajos.

\section{BIBLIOGRAFÍA}

Alvares, Z. (Febrero de 2016). Buffet don Juan . (L. A. Juárez, Entrevistador)

Díaz, I., \& Garrido, I. (15 de 5 de 2015). Correspondencias Multiples en SPSS. Recuperado el 9 de Julio de 2016, de u-cursos.cl: https://www.ucursos.cl/facso/2015/1/SO01023/1/material_ docente/bajar?id_material=1061586.

Díaz, T., \& Sánchez, G. (5 de julio de 2011). El Observador Económico. Recuperado el 22 de Septiembre de 2015, de http://www. elobservadoreconomico.com/articulo/1146

Gutiérrez, C. R. (Marzo de 2016). Comedor Cándida. (L. A. Juárez, Entrevistador)

Hernández Sampieri, D., Collado, D., \& Baptista Lucio, D. (2010). Metodología de la investigación (Quinta edición ed.). México: Mc Graw Hill.

Lovelock, C., \& Wirtz, J. (2009). Marketing de servicio. Personal, tecnología y estrategia. (P. M. Stevens, Ed., \& L. E. Ayala, Trad.) Mexico: Pearson Printice Hall. Recuperado el 5 de Octubre de 2015

Orozco, R. E. (Marzo de 2016). Buffet Estelí. (L. A. Juárez, Entrevistador)

Reyes, J. R. (Marzo de 2016). Buffet Chaman. (L. A. Juárez, Entrevistador)

Ruiz, D. O. (Abril de 2016). Buffet Sazón norteño. (L. A. Juárez, Entrevistador)

Sevilla, A. J. (Marzo de 2016). Castillo's buffet. (L. A. Juárez, Entrevistador)

Sin , autor. (3 de Mayo de 2013). El nuevo diaro. Recuperado el 27 de Julio de 2015, de El nuevo diario: http://www.elnuevodiario.com.ni/ economia/284929-nicaragua-existen-121-919empresas/ 